\title{
Aeronotificaciones especiales (ARS): colaboración ENAIRE-AEMET e importancia de la vigilancia de área
}

José Manuel López Pérez ${ }^{1}$ (jlopezp@aemet.es)

${ }^{1}$ AEMET / Delegación Territorial en la Comunidad Valenciana

\section{RESUMEN}

El Anexo 3 al Convenio sobre Aviación Civil Internacional, que recoge las normas y métodos recomendados internacionales para el servicio meteorológico para la navegación aérea internacional, dispone dos tipos de observaciones meteorológicas a bordo de aeronaves: ordinarias en las fases en ruta y ascenso inicial de vuelo (solo para aquellas equipadas con enlace de datos aire-tierra) y especiales durante cualquier fase del vuelo. Estas últimas se harán cuando las aeronaves se encuentren o se observen las siguientes condiciones: turbulencia moderada a fuerte, engelamiento moderado a fuerte, onda orográfica fuerte, tormentas con o sin granizo que se encuentren oscurecidas, inmersas generalizadas o en líneas de turbonada, tempestades de polvo o de arena fuertes, nubes de cenizas volcánicas o actividad volcánica precursora de erupción volcánica o una erupción volcánica.

El engelamiento, la turbulencia y, en gran medida, la cizalladura del viento son elementos que por el momento no pueden observarse satisfactoriamente desde tierra y respecto a los cuales en la mayoría de los casos, las observaciones de aeronave constituyen la única evidencia disponible.

Desde 2014 AEMET tiene firmado con ENAIRE un procedimiento de coordinación para la transmisión de aeronotificaciones especiales (ARS) recibidas de las aeronaves en vuelo en los centros de control de área de ENAIRE (ACC). Estas se retransmiten, en el tiempo más breve posible, mediante un formulario adjunto a un correo electrónico a las Oficinas de Vigilancia Meteorológica (OVM) de Canarias y Valencia. Con estas notificaciones, una vez validadas, las OVM codificarán un AIREP especial con difusión internacional. Si el fenómeno comunicado no dispone del aviso SIGMET correspondiente y el predictor prevé que se mantendrá durante un tiempo se elaborará un SIGMET para notificar la observación, o bien si no estuviese previsto se hará el correspondiente aviso AIRMET.

Se analizan las aeronotificaciones especiales recibidas en este periodo, su evolución y características más relevantes.

PALABRAS CLAVE: aeronotificaciones especiales; turbulencia; engelamiento; avisos de área; ENAIRE. 
